\title{
Enhancement of Chemotactic Response to Sodium Acetate in the Nematode Caenorhabditis elegans
}

\author{
Tetsuya Matsuura*, Takayuki Oda, Genta Hayashi, \\ Daisuke Sugisaki and Mitsuyuki Ichinose \\ Department of Welfare Engineering, Faculty of Engineering, \\ Iwate University, Morioka 020-8551, Japan
}

\begin{abstract}
In this study, we investigated the chemotactic response of a wild-type (N2) nematode (Caenorhabditis elegans) to a water-soluble attractant, sodium acetate, after pre-exposure to the chemical. The chemotactic response to $1.0 \mathrm{M}$ sodium acetate of the non-exposed control nematodes was lower than that of the nematodes that were pre-exposed to $1.0 \mathrm{M}$ sodium acetate for $90 \mathrm{~min}(p<0.05)$. The increase in the response to sodium acetate was observed up to $6 \mathrm{hr}$, but not at $12 \mathrm{hr}$ after exposure. To clarify the mechanism of this enhancement of the chemotactic response, several mutants were used. The chemotactic response of pre-exposed tph-1 and bas-1 mutants, whose main defect was serotonin secretion, was enhanced in comparison with that of the control mutants $(p<0.01)$. However, cat-1 and cat-2 mutants, which are respectively defective in serotonin and dopamine secretion and dopamine secretion only, showed no enhancement of the chemotactic response to sodium acetate, even when pre-exposed to this chemical. When the cat-1 and cat-2 mutants were pre-exposed to sodium acetate and bred in the presence of $40 \mathrm{mM}$ dopamine, these mutants showed enhanced chemotactic response to sodium acetate $(p<0.05)$. These results suggest that the enhancement of chemotactic response to sodium acetate after pre-exposure to this chemical is modulated by dopaminergic neurotransmission.
\end{abstract}

Key words: chemotaxis, dopamine, nematode, sensitization, sodium acetate

\section{INTRODUCTION}

The nematode Caenorhabditis elegans is a well-studied genetic model organism whose cell lineage, neuronal network, and genomic sequence have already been described (Sulston et al., 1983; White et al., 1986). C. elegans lives in soil and at air-water interfaces and feeds on soil bacteria (Andrew and Nicholas, 1976). It shows chemotactic response to various chemicals such as water-soluble substrates (e.g., $\mathrm{NaCl}$ and lysine), volatile odorants (e.g., benzaldehyde and isoamyl alcohol) and amino acids (Ward, 1973; Bargmann and Horvitz, 1991; Bargmann et al., 1993), which are produced by soil bacteria and act as natural chemotactic attractants.

C. elegans shows learned behaviors in both nonassociative (Rankin et al., 1990; Wen et al., 1997) and associative (Wen et al., 1997) learning paradigms. They recognize the association between food quantity and some chemicals. For example, when animals are maintained in the presence of $\mathrm{NaCl}$ with food bacteria, the chemotactic response to $\mathrm{NaCl}$ is enhanced in comparison with that of naive animals, whereas the chemotactic response to $\mathrm{NaCl}$ is suppressed in comparison with that of naive animals maintained in the presence of $\mathrm{NaCl}$ without food (Saeki et

\footnotetext{
* Corresponding author. Phone: +81-19-621-6376; Fax : +81-19-621-6376; E-mail: matsuura@iwate-u.ac.jp
}

al., 2001). This learning paradigm, which is associated with the presence of $\mathrm{NaCl}$ and the absence of food, has been suppressed with the addition of serotonin and/or dopamine as a neurotransmitter in food signaling (Saeki et al., 2001; Nuttley et al., 2002; Hukema et al., 2008). The insulin-like signaling pathway is also involved in salt chemotaxis learning (Tomioka et al., 2006). In contrast, non-associative learning processes such as sensitization, habituation, and dishabituation involve behavioral modification mediated by the presentation of a single cue (Carew and Sahley, 1986; Brown, 1998). In C. elegans, the continuous presentation of chemical and mechanical stimuli causes a decrease in the level of response to the same stimuli (Bargmann et al., 1993; Colbert and Bargmann, 1995; Rankin, 2000; Matsuura et al., 2009).

In our preliminary studies, we unexpectedly found that C. elegans pre-exposed to sodium acetate in the agar plate, which was obtained by a concentration gradient of sodium acetate, showed an increase in the chemotactic response to sodium acetate in comparison with naive animals (Matsuura et al., 2007b). The results of our preliminary studies were not consistent with those in previous reports (Wen et al., 1997; Saeki at al., 2001), presumably due to differences in the methods used in the conditioning period. In these previous reports, animals were soaked in an attractant solution during the conditioning period. Our conditioning method used gradients in agar plates, which more closely approximates natural conditions. From the results of our preliminary studies, we consider that the increase in the chemotactic 
response to sodium acetate may be linked to sensitization to this chemical attractant. Sensitization shows an increase in the strength of any reflex that is caused by one or more strong stimuli, usually evoked the reflex. There is no report, to the best of our knowledge, demonstrating that $C$. elegans undergoes sensitization to chemicals. Such sensitization has been extensively investigated in the sea hare Aplysia californica (Kandel, 2001). The gill-withdrawal reflex in Aplysia is habituated by repeated siphon stimuli. After an aversive shock has been applied to another part of the body, e.g., the tail, the same weak-touch stimulus increases both the size and the duration of the gill-withdrawal reflex response.

In the present study, we investigated the chemotactic response to sodium acetate of $C$. elegans pre-exposed to the same concentration of sodium acetate. Recently, it has been revealed that the chemotactic response to sodium acetate was a combination of attraction to sodium ion and acetate (Frøkjær-Jensen et al., 2008). This suggests that sodium acetate is not detected as a sodium ion-specific stimulus (Frøkjær-Jensen et al., 2008), although sodium acetate has been used as a surrogate sodium ion-specific stimulus in chemotaxis assays (e.g., Bargmann and Horvitz, 1991; Saeki et al., 2001; Matsuura et al., 2004). The aim of this study is to determine how long the retention time of the enhancement of the chemotactic response to sodium acetate continues after pre-exposure to sodium acetate using original methods that do not use anesthesia for animals at the attractant location, and to clarify the mechanism of the enhancement of chemotactic response.

\section{MATERIAL AND METHODS}

\section{Animals}

Caenorhabditis elegans wild-type strain (Bristol N2), and cat-1 (e1111), cat-1 (ok411), cat-2 (e1112), tph-1 (mg280), bas-1 (ad446) and bas-1 (tm351) mutants were obtained from the Caenorhabditis Genetics Center of the University of Minnesota. The animals were grown and maintained on nematode growth medium (NGM) agar plates $(3 \mathrm{~g} / \mathrm{l} \mathrm{NaCl}, 2.5 \mathrm{~g} / \mathrm{l}$ polypeptone, $5 \mathrm{mg} / \mathrm{l}$ cholesterol, $1 \mathrm{mM} \mathrm{CaCl}, 1 \mathrm{mM} \mathrm{MgSO}$, $25 \mathrm{mM} \mathrm{KH}_{2} \mathrm{PO}_{4}$ in pH 6.0 and 17 $\mathrm{g} / \mathrm{l}$ agar) in an incubator (Mitsubishi Electric Engineering $\mathrm{CN}-25 \mathrm{~B}$ ) at $20^{\circ} \mathrm{C}$ with the Escherichia coli OP50 strain as a food source.

In all experiments, synchronously staged young adult (YA) hermaphrodites were used during the pre-exposure to sodium acetate (pre-exposure period). To obtain them, fifty gravid animals were moved to a fresh NGM plate, allowed to lay eggs for $3 \mathrm{hr}$ at $25^{\circ} \mathrm{C}$, and removed from the plate. The animals developed into YA hermaphrodites approximately $75 \mathrm{hr}$ after the removal of gravid animals at $20^{\circ} \mathrm{C}$ (Matsuura et al., 2004; 2007a; Shingai et al., 2005). Although several hundred YA animals were grown on one NGM plate using this method, there was no effect of population density on chemotaxis index (unpublished observation), suggesting that differences in the population density during developmental period do not affect chemotactic response.

\section{Pre-exposure to sodium acetate}

Tissue culture dishes of $6-\mathrm{cm}$-diameter were used as preexposure or control plates; they contained $1 \mathrm{mM} \mathrm{CaCl}_{2}, 1 \mathrm{mM}$ $\mathrm{MgSO}_{4}, 5 \mathrm{mM} \mathrm{KH}_{2} \mathrm{PO}_{4}(\mathrm{pH} \mathrm{6.0)}$ and $17 \mathrm{~g} / \mathrm{l}$ agar. To obtain a concentration gradient, $7 \mu \mathrm{l}$ of $1.0 \mathrm{M}$ sodium acetate was placed on the surface of a pre-exposure plate $15-18 \mathrm{hr}$ and $3 \mathrm{hr}$ before the start of the pre-exposure treatment. The pre-exposure and control plates were placed in an incubator at $20^{\circ} \mathrm{C}$. The control plate contained no sodium acetate.
Animals in the YA stage were washed in a 15-ml conical centrifuge tube with wash buffer $\left(1 \mathrm{mM} \mathrm{CaCl}_{2}, 1 \mathrm{mM} \mathrm{MgSO} 4,5 \mathrm{mM}\right.$ $\mathrm{KH}_{2} \mathrm{PO}_{4}$ in $\mathrm{pH} 6.0$ and $0.05 \%$ Tween 20 ) after removing them from the NGM plates with bacteria as food, and centrifuged for $20 \mathrm{~s}$ at approximately $900 \mathrm{~g}$. In the present study, NGM plates refer to the plates that included food bacteria. All animals were washed three times prior to use. Approximately 100 animals, which were collected from the same NGM culture, were placed at two locations in the preexposure and control plates using a micropipette (Fig. 1A). All animals from the two locations (approximately 200 animals) were allowed to dry, and clumped animals were gently separated with a Kimwipe. They were left to move freely in the pre-exposure or control plates for $90 \mathrm{~min}$ in a $20^{\circ} \mathrm{C}$ incubator (conditioning period). In the N2 experiment, animals were transferred and maintained on the NGM plate in a $20^{\circ} \mathrm{C}$ incubator for $0,2,6$, or $12 \mathrm{hr}$ after pre-exposure to sodium acetate. In the mutant experiments, animals were maintained on the $\mathrm{NGM}$ plate in a $20^{\circ} \mathrm{C}$ incubator for $2 \mathrm{hr}$ after exposure to sodium acetate. The animals maintained on the preexposure plates were called pre-exposed animals, and those maintained on the control plates were called control animals.

To confirm the enhancement of chemotactic response to sodium acetate of pre-exposed animals, the difference in chemotactic response to sodium acetate between pre-exposed and naive animals was also examined. In this measurement, the pre-exposed animals were exposed to sodium acetate on a pre-exposure plate for $90 \mathrm{~min}$ at $20^{\circ} \mathrm{C}$. The naive animals were maintained on an NGM plate, which included enough food bacteria to prevent starvation, during conditioning period. Animals were transferred to an assay plate after the conditioning period and were analyzed for a chemotactic response to sodium acetate.

The pre-exposed and control animals were given strong mechanical stimuli three times in the form of approximately $1800 \mathrm{~g}$ spins using a centrifuge to clarify whether the enhancement of chemotactic response to sodium acetate is non-associative or associative learning. Both groups of animals were maintained on the NGM plate in a $20^{\circ} \mathrm{C}$ incubator for $2 \mathrm{hr}$ after conditioning period. The animals of each group were washed in a 15-ml conical centrifuge tube with $10 \mathrm{ml}$ wash buffer after removing them from the NGM plates and centrifuged for $60 \mathrm{~s}$ at approximately $1800 \mathrm{~g}$ spins. For the next two times, the supernatant was removed, and more wash buffer was added to a final volume of $10 \mathrm{ml}$ prior to centrifuging at $1800 \mathrm{~g}$ for $60 \mathrm{sec}$. The chemotaxis assay was performed subsequently (see below).

\section{Treatment of serotonin or dopamine}

For treatment with serotonin or dopamine, the mutants were exposed during periods of pre-exposure to sodium acetate and maintenance on the NGM plate (Fig. 1B). In the case of serotonin treatment, $200 \mu \mathrm{l}$ of $0.1 \mathrm{M}$ serotonin was spread on the preexposure, control, and NGM plates. In the case of dopamine treatment, dopamine was dissolved in the pre-exposure, control, and NGM plates at a final concentration of $40 \mathrm{mM}$. The efficacy of the concentrations of serotonin and dopamine has been confirmed on the basis of previous reports (Nuttley et al., 2002; Sanyal et al., 2004). Approximately 100 animals were placed at two locations in the pre-exposure and control plates for $90 \mathrm{~min}$ and maintained on the NGM plate for $2 \mathrm{hr}$.

\section{Chemotaxis assay}

In the chemotaxis assay, we used 9-cm-diameter tissue culture dishes containing $1 \mathrm{mM} \mathrm{CaCl}_{2}, 1 \mathrm{mM} \mathrm{MgSO}_{4}, 5 \mathrm{mM} \mathrm{KH}_{2} \mathrm{PO}_{4}$ of $\mathrm{pH}$ 6.0 and $17 \mathrm{~g} / \mathrm{l}$ agar. For the assay, $7 \mu \mathrm{l}$ of $1.0 \mathrm{M}$ sodium acetate was spotted at the sodium acetate location (location A in Fig. 1) on each assay plate 15-18 $\mathrm{hr}$ and $3 \mathrm{hr}$ before the start of the assay to obtain a concentration gradient. As a control, $7 \mu \mathrm{l}$ of water was spotted at control location B. In this study, we did not use sodium azide as anesthesia for the animals, in order to observe the differences in 


\section{A Pre-exposed animals}

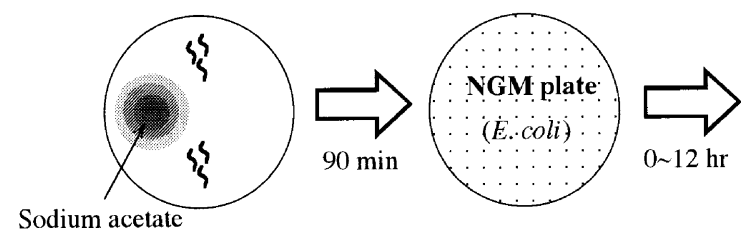

\section{Control animals}
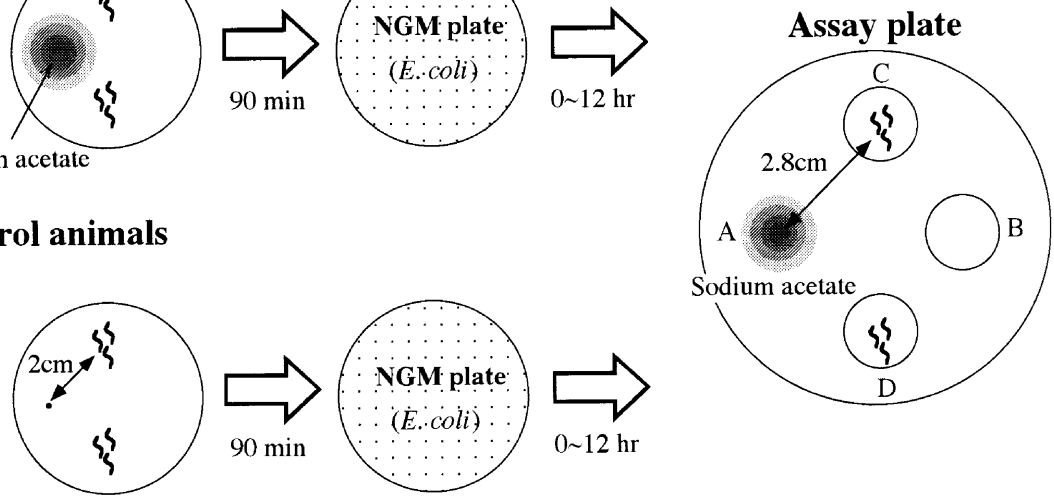

\section{B Pre-exposed animals}

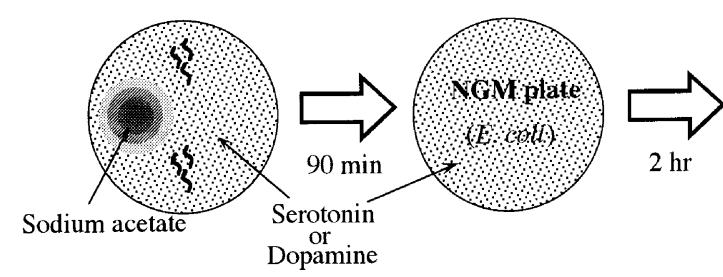

Control animals

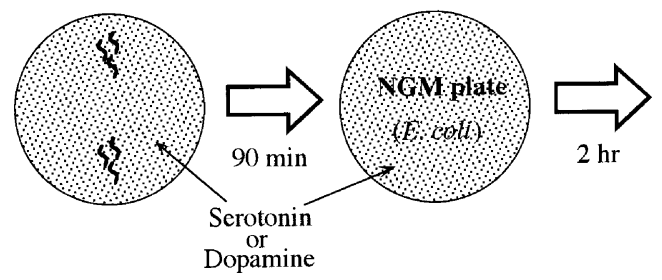

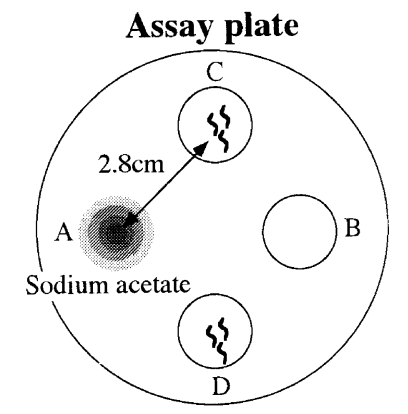

in Fig. 1). Animals at the original locations were dried and then gently separated with a Kimwipe. The numbers of animals at locations $A$ and $B$ (circle of $1 \mathrm{~cm}$ radius) were determined by microscopy (Olympus SZ40) every $10 \mathrm{~min}$. The chemotaxis assay of the pre-exposed animals was always carried out together with that of the control or naive animals to avoid any difference in the animals' conditions. The assay was performed in an experimental room maintained at $20^{\circ} \mathrm{C}$.

\section{Measurement of locomotor activity}

To determine locomotor activity of animals, we measured the spontaneous locomotor rate, which is known to be an index of the speed of locomotion. One animal was placed onto a no-attractant assay plate. After $5 \mathrm{~min}$, the number of body bends including omega turns (Duhon and Johnson, 1995) was measured for each animal during five separate 30 -sec time periods for 5 min intervals. The values of five trials were again averaged over the number of animals used. Twenty measurements were performed at the YA stage.

\section{Scoring and analysis}

The chemotaxis index was calculated on the basis of the number of animals that gathered at the sodium acetate location (Bargmann et al., 1993) using the following equation: chemotaxis index $=$ (number of animals at sodium acetate location number of animals at control location)/ (total number of animals on plate). To evaluate the chemotactic response during the assay, the integral chemotaxis index was also calculated (Matsuura et al., 2009). The index indicated the approximate total number of animals for a 90-min assay. The integral chemotaxis index evaluated sensation and habituation behaviors during the assay. A higher index indicates that animals exhibit fast gathering due to high sensitivity to an attractant.

The significance of the difference in chemotaxis index or integral chemotaxis index was analyzed using Student's $t$-test (two-sided test). The difference in time courses was statistically examined using two-way ANOVA. Values are presented as means \pm S.E.M.

\section{RESULTS}

the gathering and withdrawal behaviors at the attractant location between pre-exposed and control animals.

The pre-exposed and control N2 animals, which were maintained on NGM plates, were washed two times with the wash buffer after removing them from the plate and centrifuged for $60 \mathrm{~s}$ at approximately $450 \mathrm{~g}$. To avoid the effect of population density on chemotactic response during the assay (Matsuura et al., 2005), approximately 30 animals were placed at locations $C$ and $D$, equidistant $(2.8 \mathrm{~cm})$ from the attractant and control spots (assay plate
Comparisons of chemotactic response to sodium acetate between pre-exposed and naive animals, and between control and naive animals

The time courses showed that the chemotaxis index for sodium acetate of naive animals, which were maintained on an NGM plate during conditioning period, increased slowly over $40 \mathrm{~min}$ and then slightly decreased, whereas that of pre-exposed animals increased for $20 \mathrm{~min}$, after which they 
left the sodium acetate location (Fig. 2A). The chemotaxis indices of the pre-exposed animals increased significantly from 10 to 30 min in comparison with those of the naive animals $(p<0.01)$, and the index of the preexposed animals was significantly smaller than that of naive animals at 90 min ( $p<$ $0.01)$. The integral chemotaxis index of the pre-exposed animals was larger than that of naive animals $(p<$ 0.05) (Fig. 2B). Moreover, there was no significant difference in the levels of spontaneous activity between pre-exposed and naive animals (Fig. 5), indicating that the enhancement of the chemotactic response to sodium acetate in preexposed animals was not caused by an increase in locomotor activity.

The chemotaxis indices of control animals increased significantly from 10 to $20 \mathrm{~min}(p<0.01)$ and decreased from 60 to $90 \mathrm{~min}$ in comparison with those of the naive animals ( $p<0.05$ at $60 \mathrm{~min} ; p<0.01$ at $90 \mathrm{~min}$ ) (Fig. 2A). Therefore, we did not observe a significant difference in the integral chemotaxis index between control and naive animals (Fig. 2B). An enhancement of chemotactic response in the control animals during the early period of the assay indicates that the enhancement of the chemotactic response is caused by not only pre-exposure to sodium acetate, but also by starvation during the pre-exposure period (see discussion).

\section{Retention time of the enhancement of the chemotactic response to sodium acetate}

To confirm the retention time of the increase in the chemotactic response to sodium acetate in pre-exposed animals, we compared the time course of chemotaxis indices for $1.0 \mathrm{M}$ sodium acetate between pre-exposed and control animals. The time courses of the two groups showed that the number of animals attracted to sodium acetate was higher than that of animals that left the area with the first 30 min (Fig. 2A). When animals were maintained on the NGM plate for $6 \mathrm{hr}$, the chemotaxis indices of the pre-exposed animals were larger than those of the control ones $(p<$ 0.05 ). However, no significant difference in the time course of chemotaxis indices between pre-exposed and control ani-
A

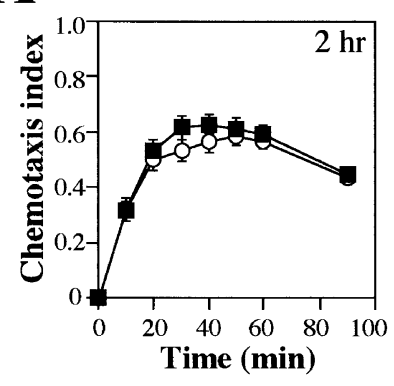

B

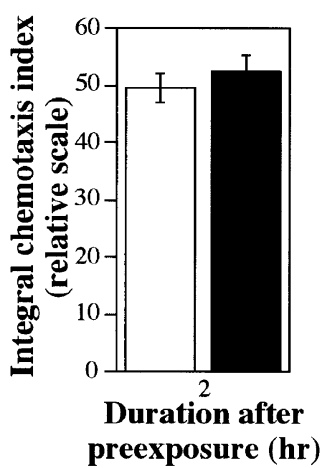

Fig. 3. (A) Time courses of changes in average chemotaxis index for $1.0 \mathrm{M}$ sodium acetate of pre-exposed and control animals, which were maintained on the NGM plate for $2 \mathrm{hr}$ and exposed to strong mechanical stimuli. Note that no enhancement of response level to 1.0 $\mathrm{M}$ sodium acetate was observed in the pre-exposed animals, suggesting that the enhancement of the chemotactic response during the assay may be linked to sensitization to sodium acetate. Open circles and closed squares indicate the mean chemotaxis indices of control and pre-exposed animals, respectively. (B) Integral chemotaxis index for $1.0 \mathrm{M}$ sodium acetate. White and black columns are the integral chemotaxis indices of the control and preexposed animals, respectively. Error bars indicate S.E.M. $(n \geq 22$ assays). 
mals was found when animals were maintained on the NGM plate for $12 \mathrm{hr}$. The integral chemotaxis index of the preexposed animals was also larger than that of control animals at $6 \mathrm{hr}(p<0.05)$, but not at $12 \mathrm{hr}$ (Fig. 2B), indicating that the effect of sodium acetate on the enhancement of the response to this chemical was maintained beyond $6 \mathrm{hr}$, but not up to $12 \mathrm{hr}$. These results suggest that preexposed animals show a high sensitivity and gather more readily at the sodium acetate location in comparison with control animals.

There were differences in the duration of the maintenance period on the NGM plate (Fig. 2A) in the chemotaxis indices of time courses. Moreover, the integral chemotaxis index became larger with an increase in the maintenance period (Fig. 2B). It has been reported that the chemotaxis indices for sodium acetate in $\mathrm{N} 2$ animals varied by developmental stage (Matsuura et al., 2007a). Therefore, the higher chemotaxis indices of $6 \mathrm{hr}$ and $12 \mathrm{hr}$ may have been caused by changes in sensitivity to sodium acetate over the course of development. Differences in experimental and/or animal conditions may be one of the reasons for the differences in chemotaxis indices.

When the pre-exposed animals were given strong mechanical stimuli (i.e., three times centrifuging at $1800 \mathrm{~g}$ for $60 \mathrm{sec}$ ), no enhancement of the response level to $1.0 \mathrm{M}$ sodium acetate was observed in the pre-exposed animals (Fig. 3). It is well known that $C$. elegans recover from the non-associative learning following exposure to other strong stimuli, such as mechanical stimulation (Broster and Rankin, 1994; Wicks and Rankin, 1996). The result indicates that the enhancement of the response to sodium acetate in the preexposed animals is not caused by associative learning, and suggests that the phenomenon during the assay is sensitization to sodium acetate due to pre-exposure to sodium acetate. The time courses showed that the leaving behavior of the two groups of animals, which were given strong mechanical stimuli, for sodium acetate were slower than those of animals that were not exposed to such stimuli (Figs. $2 \mathrm{~A}$ and $3 \mathrm{~A}$ ). It is possible that the slow leaving behavior is caused by a change in some condition in the animals due to strong mechanical stimuli. cate S.E.M. $\dagger: p<0.01$ ( $n \geq 12$ assays).
Dopamine is associated with the enhancement of the chemotactic response to sodium acetate

As mentioned above, starvation is one of the factors that enhances the chemotactic response to sodium acetate (Fig.

Fig. 4. Association of serotonin or dopamine secretion system with the enhancement of the chemotactic response to $1.0 \mathrm{M}$ sodium acetate. (A) Time courses of changes in average chemotaxis index for $1.0 \mathrm{M}$ sodium

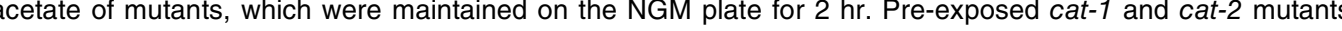
作 (1,7) $=27.49, \mathrm{p}<0.01$; bas-1 $F(1,7)=18.22, \mathrm{p}<0.01)$. Open circles and closed squares indicate the indices of the control and pre-exposed mutants, respectively. (B) Integral chemotaxis index exposed tph-1 and bas-1 mutants in contrast were larger than those of control mutants. White and black columns show the integral chemotaxis indices of control and pre-exposed animals, respectively. Error bars indi-

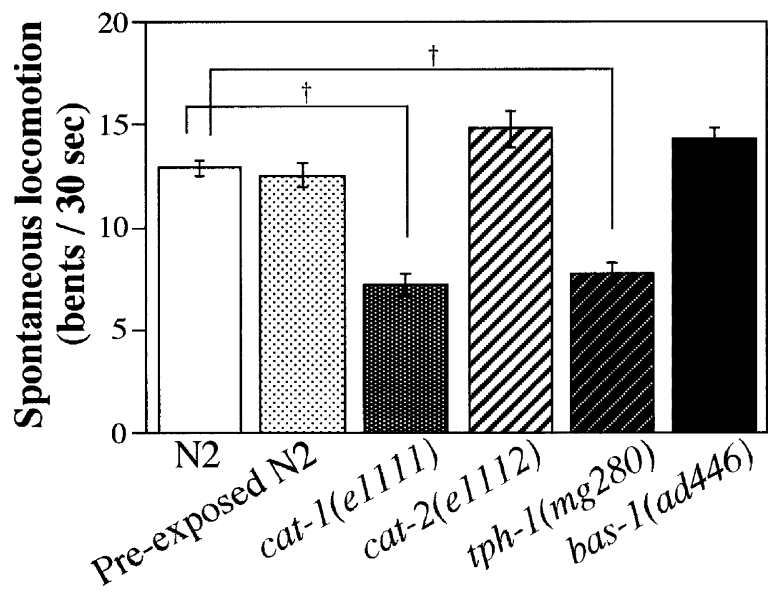

Fig. 5. Locomotor activity of wild-type animals and some mutants. The number of body bends (spontaneous activity) was measured for each animal during five separate 30-sec time periods for 5 min intervals. The values of five trials were again averaged over the number of animals used $(n=20)$. The levels of spontaneous activity of the cat-1 and tph-1 mutants were significantly lower than those of N2 animals $(\dagger: p<0.01)$, and there was no significant difference in the level of spontaneous activity between pre-exposed N2 and naive (N2) animals. 
2). This supposes that the enhancement of the chemotactic response after pre-exposure to sodium acetate may be associated with a substrate mediated by food signaling and insulin-like signaling pathways, such as serotonin and dopamine (Saeki et al., 2001; Sanyal et al., 2004; Tomioka et al., 2006). To confirm whether the enhancement of the chemotactic response to sodium acetate was caused by serotonin and/or dopamine, we investigated the chemotactic response using mutants with defects in the secretion of serotonin and/or dopamine.

Pre-exposed cat-1 and cat-2 mutants showed no enhancement of chemotactic response to sodium acetate. The time courses of the pre-exposed and control cat- 1 mutants showed that the animals were attracted to sodium acetate up to $40 \mathrm{~min}$ and then left the sodium acetate location, whereas the chemotaxis index of the two groups of cat-2 mutants increased up to $60 \mathrm{~min}$ and decreased slightly after 60 min (Fig. 4A). In the cat-1 and cat-2 mutants, there was no significant difference between the pre-exposed and control mutants in the chemotaxis index at each time point. The integral chemotaxis index of the pre-exposed mutants showed almost the same value as that of the control mutants (Fig. 4B). Preexposed tph-1 and bas-1 mutants in contrast showed an enhancement of the chemotactic response to sodium acetate in comparison with control mutants $(p<0.01)$ (Fig. 4A). The integral chemotaxis indices of the pre-exposed tph-1 and bas-1 mutants were also larger than those of the control mutants $(p<0.01)$ (Fig. 4B). The cat-1 mutants showed a defect in the secretions of both serotonin and dopamine (Duerr et al., 1999; Sawin et al., 2000). The tph-1 and bas-1 mutants may have a deficiency mainly in serotonin secretion (see discussion) (Loer and Kenyon, 1993; Murakami et al., 2008), while the cat-2 mutants show a defect in the secretion of dopamine (Loer and Kenyon, 1993; Sawin et al., 2000). Our observations suggest that the secretion of dopamine is associated with the enhancement of the chemotactic response to sodium acetate after pre-exposure to this chemical.

The chemotaxis indices of the cat-1 and tph-1 mutants were lower than those of $\mathrm{N} 2$ and other animals (Figs. 2 and 4). The levels of spontaneous activity in the cat-1 and tph-1 mutants were also significantly lower than those in $\mathrm{N} 2$ animals and other mutants $(p<0.01)$ (Fig. 5). There was no obvious difference in spontaneous locomotion among N2 animals, cat-2 and bas-1 mutants (Fig.
5), consistent with previous reports (Loer and Kenyon, 1993; Sawin et al., 2000), although serotonin and/or dopamine signaling affects the locomotor speed of animals (Hobert, 2003; Sanyal et al., 2004). This indicates that the differences in the time course among N2 animals, cat-2 and bas- 1 mutants are not caused by a difference in locomotor speed. These results suggest that the slow response to sodium acetate of cat-2 and bas-1 mutants is caused by low sensitivity to this chemical, and that the slow movement of the cat-1 and tph1 mutants causes low chemotactic response to sodium acetate of these mutants.

The pre-exposed cat-1 mutants in the presence of serotonin showed no enhancement of the chemotactic response to sodium acetate (Fig. 6A). There was no significant difference in integral chemotaxis index between the pre-exposed and control cat-1 mutants (Fig. 6C). When the cat-1 mutants were maintained in the presence of dopamine, the time courses of the pre-exposed and control mutants increased

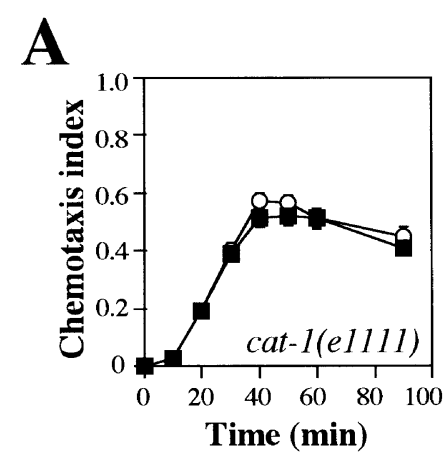

B
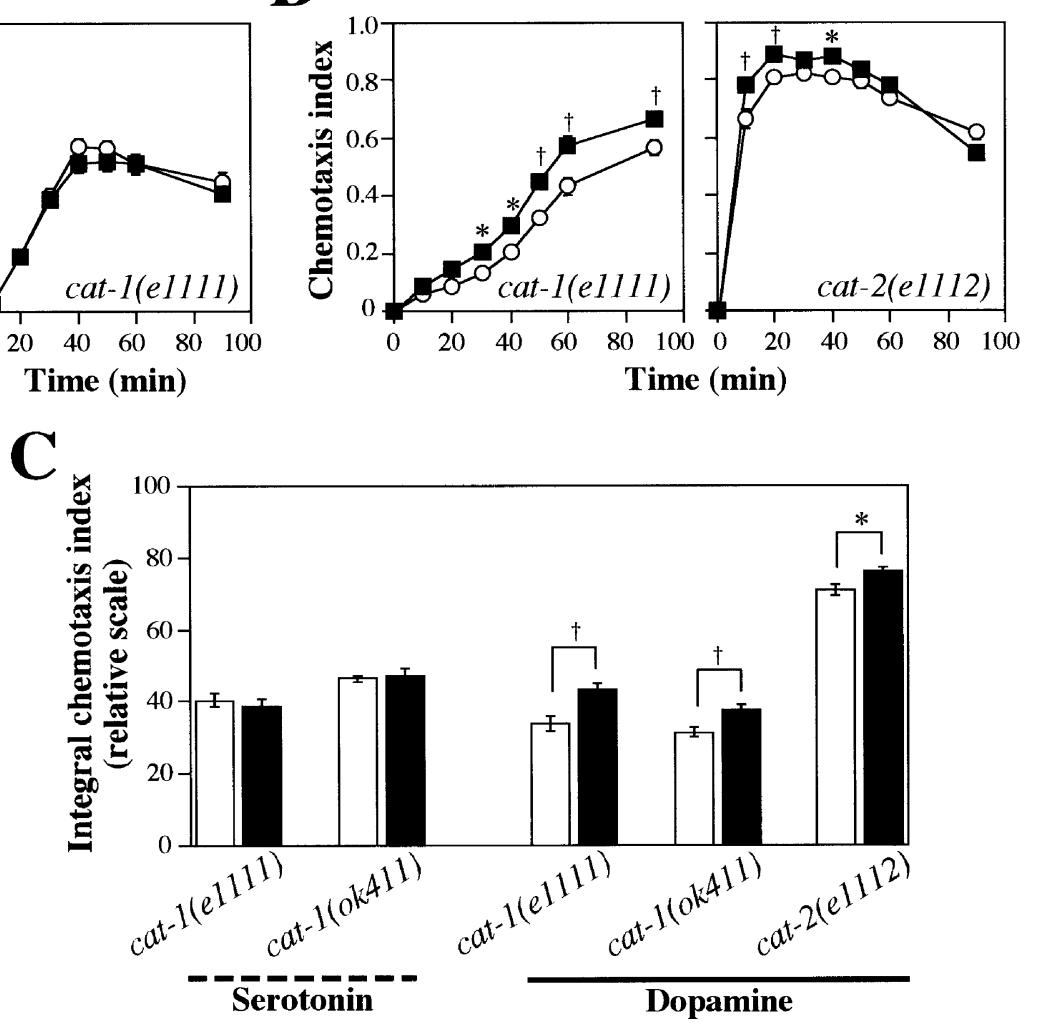

Fig. 6. Effect of serotonin or dopamine treatment on the chemotactic response to $1.0 \mathrm{M}$ sodium acetate in cat-1 and cat-2 mutants. (A) Time courses of changes in the average chemotaxis index for $1.0 \mathrm{M}$ sodium acetate of cat-1 mutants, which were maintained on the pre-exposure or control plate for $90 \mathrm{~min}$ and the NGM plate for $2 \mathrm{hr}$ in the presence of serotonin. (B) Time courses of changes in the average chemotaxis index for $1.0 \mathrm{M}$ sodium acetate of cat-1 and cat-2 mutants, which were maintained on the pre-exposure or control plate for 90 min and the NGM plate for $2 \mathrm{hr}$ under the condition of the presence of dopamine (cat-1 $F(1,7)=19.45, p<0.01$; cat-2 $F(1,7)=11.03, p<0.05)$. Open circles and closed squares indicate the mean chemotaxis indices of the control and pre-exposed mutants, respectively. (C) Integral chemotaxis index for $1.0 \mathrm{M}$ sodium acetate. After the presence of dopamine, the integral chemotaxis indices of preexposed cat-1 and cat-2 mutants were larger than those of control mutants. White and black columns show the integral chemotaxis indices of control and pre-exposed animals, respectively. Error bars indicate S.E.M. $\dagger: p<0.01, *: p<0.05$ ( $n \geq 10$ assays). See details in the text. 
with an increase in the time of the assay for $90 \mathrm{~min}$ (Fig. $6 \mathrm{~B})$. The time course showed that the chemotaxis indices of the cat-1 and cat-2 mutants pre-exposed to sodium acetate with dopamine were larger than those of the control mutants at some time points (Fig. 6B). Increases in the integral chemotaxis index for sodium acetate of the pre-exposed cat- 1 and cat- 2 mutants were also observed $(p<0.01$ for cat-1 mutants; $p<0.05$ for cat-2 mutants) (Fig. $6 \mathrm{C}$ ).

\section{DISCUSSION}

Some of the chemotaxis indices of the pre-exposed animals were larger than those of the control ones $(p<0.01)$, and the indices of the control animals were also enhanced significantly from 10 to $20 \mathrm{~min}$ in comparison with those of the naive animals $(p<0.01)$ (Fig. 2A upper left panel). Therefore, we cannot ignore the effect of starvation and other factors during the conditioning period on the enhancement of the chemotactic response of pre-exposed animals. However, in our experimental procedure, the pre-exposed and control animals experienced the starvation for the same duration during conditioning period, and both groups of animals were maintained under the same conditions, e.g., food, temperature, animal density, after the conditioning period. The only difference in the experimental condition between the pre-exposed and control animals was the presence or absence of sodium acetate during conditioning period (Fig. $1 \mathrm{~A})$. Moreover, the chemotaxis indices of the pre-exposed animals were larger than those of the control ones when animals were maintained on the NGM plate for $6 \mathrm{hr}(p<0.05)$ (Fig. 2). We concluded that some part of the enhancement was caused by pre-exposure to sodium acetate, and thus the term of "enhancement" was used in the present paper.

Sodium azide had been used to anesthetize animals in conventional assays. When sodium azide is spotted on the attractant location of the assay plate, the leaving behavior of animals during chemotactic assay cannot be observed (Matsuura et al., 2009). Therefore, we did not use sodium azide to observe the behavior of $C$. elegans under natural conditions in the present study. The time courses revealed that the peak values of chemotaxis indices in pre-exposed animals were higher than those in control animals, and that the ratio of leaving behavior of pre-exposed animals from sodium acetate location was higher than that of control ones (e.g., Fig. 2), except for the time courses of the responses to sodium acetate of tph-1 and dopamine-treated cat-1 mutants (Figs. 4A and $6 \mathrm{~B}$ ). These results suggest that the sensitivity to sodium acetate of pre-exposed animals was enhanced, but attenuated earlier than that of the control animals.

C. elegans recognizes the association between the absence of food and the presence of $\mathrm{NaCl}$. When $C$. elegans was maintained under the condition of the presence of $\mathrm{NaCl}$ and the absence of food, the chemotactic index for $\mathrm{NaCl}$ was significantly lower than that of naive animals (Saeki et al., 2001; Nuttley et al., 2002). Serotonin is involved in this salt-associative learning as a component of food signaling and insulin-like signaling pathways (Saeki at al., 2001; Tomioka et al., 2006). We found, however, that the chemotactic response to sodium acetate of pre-exposed animals is greater than that of control animals when maintained on an agar plate with a concentration gradient of 1.0
M sodium acetate in the absence of food. The integral chemotaxis index of these animals was also higher than that of the control animals (Fig. 2). It has been reported that the recovery from non-associative learning processes, such as habituation and sensitization, was observed with other strong stimuli (Broster and Rankin, 1994; Wicks and Rankin, 1996). In the present study, no enhancement of the response level to $1.0 \mathrm{M}$ sodium acetate was observed when pre-exposed animals were given strong mechanical stimuli (Fig. 3). Moreover, pre-exposed Irn-1 mutants, which have a defect in associative learning in regards to water-soluble attractants, showed an enhancement of chemotactic response to sodium acetate (Matsuura et al., 2007b). The chemotactic response to sodium acetate in animals conditioned in the presence of food with sodium acetate was greater than that of animals maintained in the presence of food only (unpublished observation). These observations indicate strongly that the enhancement of the response to sodium acetate is a type of non-associative learning, and suggest that the enhancement of the response to sodium acetate is linked to sensitization to this chemical.

In previous reports studying learning processes, animals were soaked in $\mathrm{NaCl}$ solution duirng the pre-exposure period, and the chemotaxis index for $\mathrm{NaCl}$ was measured during the assay (Wen et al., 1997; Saeki at al., 2001; Tomioka et al., 2006). When animals were maintained in the presence of $\mathrm{NaCl}$ without food, the chemotactic response to $\mathrm{NaCl}$ was suppressed in comparison with that of naive animals (Saeki at al., 2001). In the present study, we used sodium acetate and confirmed an enhancement of chemotactic response in pre-exposed animals. Sodium acetate has been used as a surrogate for sodium ion-specific stimulus in chemotaxis assays (e.g., Bargmann and Horvitz, 1991; Saeki et al., 2001; Matsuura et al, 2004). Importantly, however, Frøkjær-Jensen et al. (2008) revealed that sodium acetate was not detected as a sodium ion-specific stimulus. It is well known that the sensitization is regarded as an increase in broader sensory response to one or more stimuli. Therefore, the enhancement of the chemotactic response to sodium acetate may be caused by increases in the sensitivity of sensory neurons, which detect sodium ion and acetate, after the experience of sodium acetate stimulation.

When the animals were maintained at $20^{\circ} \mathrm{C}$ after being pre-exposed to an odorant diacetyl, the chemotaxis index for diacetyl of the pre-exposed animals was smaller than that of the control animals up to $6 \mathrm{hr}(p<0.05)$, but not at $12 \mathrm{hr}$ (Matsuura et al., 2009). In this study, the enhancement of the chemotactic response to sodium acetate was also maintained beyond $6 \mathrm{hr}$, but not at $12 \mathrm{hr}$ (Fig. 2). We have noted that $C$. elegans shows a decrease in the chemotactic response to attractant after $2 \mathrm{hr}$ of starvation (unpublished observation). A decrease in neurotransmitter level within 30 min was also reported in $C$. elegans under the condition of food absence (Sawin et al., 2000; Saeki et al., 2001). These observations suggest that any neurotransmitter level associated with animals' behavior, such as those of serotonin and dopamine secretion, may need to recover over $6 \mathrm{hr}$ under the condition of food presence. L'Etoile et al. (2002) reported that learning behavior was initiated by intrinsic processes in the sensory neuron, e.g., phosphorylation of sig- 
naling molecules. It is a possible that $6 \mathrm{hr}$ is the limit of retention time of $C$. elegans' learning, which may be due to changes in the growth conditions of animals, such as the recovery from phosphorylation of molecules and changes in neurotransmitter release.

The genes cat-1, cat-2, tph-1 and bas-1 encode enzymes involved in serotonin and/or dopamine production, and mutations of these genes result in deficiencies in the secretion of serotonin and/or dopamine. cat-1 encodes a synaptic vesicular monoamine transporter required for the presence of dopamine and serotonin in nerve terminals; therefore, cat-1 mutants have defects in both serotonin and dopamine secretions (Duerr et al., 1999). Dopamine synthesis involves the CAT-2 tyrosine hydroxylase (Loer and Kenyon, 1993), and cat-2 mutants show a defect in dopamine secretion in the nervous system (Sawin et al., 2000). tph-1 encodes tryptophan hydroxylase, the enzyme that catalyzes the rate-limiting first step in serotonin biosynthesis, and mutations of tph-1 impair serotonin secretion (Loer and Kenyon, 1993; Sawin et al., 2000; Murakami et al., 2008). Although it is known that bas- 1 encodes a serotonin- and dopamine-synthetic aromatic amino acid decarboxylase that is required for the synthesis of serotonin from 5-hydroxytryptophan (Sawin et al., 2000), bas-1 mutants may have a deficiency mainly in serotonin secretion (Loer and Kenyon, 1993; Murakami et al., 2008). Pre-exposed cat-1 and cat-2 mutants showed no enhancement of the chemotactic response to sodium acetate, while pre-exposed tph-1 and bas-1 mutants showed an increase in chemotactic response to sodium acetate in comparison to control mutants ( $p<$ 0.01 ) (Fig. 4). These results suggest that dopamine secretion is associated with the enhancement of the chemotactic response to sodium acetate after pre-exposure to this chemical.

Moreover, the increase in the integral chemotaxis index for sodium acetate was observed in cat-1 mutants when they were pre-exposed to sodium acetate in the presence of dopamine, although there was no significant difference in the chemotactic response to sodium acetate between preexposed and control cat-1 mutants when cat-1 mutants were pre-exposed to sodium acetate in the presence of serotonin (Fig. 6). When cat-2 mutants were pre-exposed to sodium acetate in the presence of dopamine, the chemotaxis index of the pre-exposed cat-2 mutants was also larger than that of control mutants (Fig. 6). Dopamine receptor stimulation plays a central role in behavioral plasticity, in that it enhances sensitization (Sanyal et al., 2004). In the experiment of dopamine treatment, the time courses and the levels of difference in the chemotaxis index between preexposed and control mutants were not comparable to those of N2 animals (Figs. 2 and 6). The experimental conditions of pre-exposed mutants, e.g., duration of dopamine treatment, maintenance period of NGM plate, were the same as those of control mutants (Fig. 1B), indicating that the efficacies of dopamine to the pre-exposed and control mutants should be identical. This suggests that the dopamine treatment affects not only the locomotor speed (see below) but also causes an increase in the chemotactic response of animals, and enhances the response to sodium acetate in preexposed mutants. These observations support the possibility that dopamine is an important factor in sensitization to sodium acetate in $C$. elegans.

There are differences in the time course between the animals that were treated with serotonin or dopamine and those that were not. For example, the chemotaxis indices of cat-1 mutants exposed to serotonin were higher than those of animals not treated with serotonin (Figs. 4A and 6A). The role of serotonin extends far beyond the control of locomotor speed, and is a critical molecular device employed in a specific sensory context to modulate the activity of a large set of distinct neuronal circuits (Hobert, 2003). An increase in the chemotactic response to sodium acetate after serotonin treatment is caused by an increase in locomotor activity. Moreover, the time course of the chemotaxis index of the cat-1 mutants after the addition of dopamine gradually increased during the 90-min assay (Fig. 6B), whereas that of animals not treated with dopamine peaked within 40 min (Fig. 4A). The dopamine system in the central nervous system plays a critical role in motor control, rewards, and cognition (Sanyal et al., 2004), suggesting that the differences in the time course between non-treated and dopamine-treated animals are caused by changes in locomotor activity.

As mentioned above, $C$. elegans feeds on soil bacteria (Andrew and Nicholas, 1976), which produce small amounts of chemicals such as volatile odorants (e.g., alcohol and diacetyl) and water-soluble substrates (e.g., the sodium ion, chloride ion and (AMP) as metabolic by-products. These chemicals are natural chemotactic attractants for nematodes. In nature, C. elegans encounters water-soluble attractants at specific concentrations, indicating that the presence of food within their vicinity. If they were to have enhanced sensitivity to attractants, the possibility of them getting food would be greater than that of ordinary animals, which have a low sensitivity to chemicals. Sensitization to water-soluble substrates is needed as a survival strategy for C. elegans in nature. The findings in this study are important for a better understanding of the plasticity of the neuronal system, such as the mechanisms of learning and memory.

\section{ACKNOWLEDGMENTS}

The nematode strains were provided by the Caenorhabditis Genetics Center. This work was partially supported by a grant for scientific research to T. M. from the Japanese Ministry of Education, Culture, Sports, Science and Technology.

\section{REFERENCES}

Andrew PA, Nicholas WL (1976) Effect of bacteria on dispersal of Caenorhabditis elegans (Rhabditiae). Nematologica 22: 451461

Bargmann Cl, Horvitz HR (1991) Chemosensory neurons with overlapping functions direct chemotaxis to multiple chemicals in $C$. elegans. Neuron 7: 729-742

Bargmann Cl, Thomas JH, Horvitz HR (1990) Chemosensory cell function in the behavior and development of Caenorhabditis elegans. Cold Spring Harbor Symp Quant Biol 55: 529-538

Bargmann Cl, Hartwieg E, Horvitz HR (1993) Odorant-selective genes and neurons mediate olfaction in $C$. elegans. Cell 74: 515-527

Broster BS, Rankin CH (1994) Effects of changing interstimulus interval during habituation in Caenorhabditis elegans. Behav Neurosci 108: 1019-1029

Brown GD (1998) Operational terminology for stimulus exposure (SE) conditioning. Behav Brain Res 95: 143-150 
Carew TJ, Sahley CI (1986) Invertebrate learning and memory: From behavior to molecules. Annu Rev Neurosci 9: 435-487

Colbert HA, Bargmann Cl (1995) Odorant-specific adaptation pathways generate olfactory plasticity in C. elegans. Neuron 14: 803-812

Duerr JS, Frisby DL, Gaskin J, Duke A, Asermely K, Huddleston D, Eiden LE, Rand JB (1999) The cat-1 gene of Caenorhabditis elegans encodes a vesicular monoamine transporter required for specific monoamine-dependent behaviors. J Neurosci 19: 72-84

Duhon S, Johnson TE (1995) Movement as an index of vitality: comparing wild type and age-1 mutant of Caenorhabditis elegans. J Gerontol 50A: B254-B261

Frøkær-Jensen C, Ailion M, Lockery SR (2008) Ammonium-acetate is sensed by gustatory and olfactory neurons in Caenorhabditis elegans. PLoS ONE 3: 1-9 (e2467)

Hobert O (2003) Behavioral plasticity in C. elegans: paradigms, circuits, genes. J Neurobiol 54: 203-223

Hukema RK, Rademakers S, Jansen G (2008) Gustatory plasticity in $C$. elegans involves integration of negative cues and $\mathrm{NaCl}$ taste mediated by serotonin, dopamine, and glutamate. Learn Mem 15: 829-836

Kandel ER (2001) The molecular biology of memory storage: a dialogue between genes and synapses. Science 294: 1030-1038

L'Etoile ND, Coburn CM, Eastham J, Kistler A, Gallegos G, Bargmann $\mathrm{Cl}$ (2002) The cyclic GMP-dependent protein kinase EGL-4 regulates olfactory adaptation in C. elegans. Neuron 36 : 1079-1089

Loer CM, Kenyon CJ (1993) Serotonin-deficient mutants and male mating behavior in the nematode Caenorhabditis elegans. J Neurosci 13: 5407-5417

Matsuura T, Oikawa T, Wakabayashi T, Shingai R (2004) Effect of simultaneous presentation of multiple attractants on chemotactic response of the nematode Caenorhabditis elegans. Neurosci Res 48: 419-429

Matsuura T, Sato T, Shingai R (2005) Interactions between Caenorhabditis elegans individuals during chemotactic response. Zool Sci 22: 1095-1103

Matsuura T, Endo S, Iwamoto R, Takahashi H, Ichinose M (2007a) Developmental changes in chemotactic response and choice of two attractants, sodium acetate and diacetyl, in the nematode Caenorhabditis elegans. Comp Biochem Physiol A 147: 920927

Matsuura T, Oda T, Hayashi G, Sugisaki D, Ichinose M (2007b) Sensitization to water-soluble sodium acetate in the nematode Caenorhabditis elegans. Comp Biochem Physiol B 148: 345

Matsuura T, Suzuki S, Musashino A, Kanno R, Ichinose M (2009) Retention time of attenuated response to diacetyl after preexposure to diacetyl in Caenorhabditis elegans. J Exp Zool 311A: 483-495

Murakami H, Bessinger K, Hellmann J, Murakami S (2008) Manipu- lation of serotonin signal suppresses early phase of behavioral aging in Caenorhabditis elegans. Neurobiol Aging 29: 10931100

Nuttley WM, Atkinson-Leadbeater KP, van der Kooy D (2002) Serotonin mediates food-odor associative learning in the nematode Caenorhabditis elegans. Proc Natl Acad Sci USA 99: 1244912454

Rankin CH, Beck CDO, Chiba CM (1990) Caenorhabditis elegans: A new model system for the study of learning and memory. Behav Brain Res 37: 89-92

Saeki S, Yamamoto M, lino Y (2001) Plasticity of chemotaxis revealed by paired presentation of a chemoattractant and starvation in the nematode Caenorhabditis elegans. J Exp Biol 204: $1757-1764$

Sanyal S, Wintle RF, Kindt KS, Nuttley WM, Arvan R, Fitzmaurice P. et al. (2004) Dopamine modulates the plasticity of mechanosensory responses in Caenorhabditis elegans. EMBO J 23: 473-482

Sawin ER, Ranganathan R, Horvitz HR (2000) C. elegans locomotory rate is modulated by the environment through a dopaminergic pathway and by experience through a serotonergic pathway. Neuron 26: 619-631

Shingai R, Wakabayashi T, Sakata K, Matsuura T (2005) Chemotaxis of Caenorhabditis elegans during simultaneous presentation of two water-soluble attractants, L-lysine and chloride ions. Comp Biochem Physiol A 142: 308-317

Sulston JE, Schierenberg E, White JG, Thomson JN (1983) The embryonic cell lineage of the nematode Caenorhabditis elegans. Dev Biol 100: 64-119

Tomioka M, Adachi T, Suzuki H, Kunitomo H, Schafer W, lino $\mathrm{Y}$ (2006) The insulin/PI 3-kinase pathway regulates salt chemotaxis learning in Caenorhabditis elegans. Neuron 51: 613625

Ward S (1973) Chemotaxis in the nematode Caenorhabditis elegans: identification of attractants and analysis of the response by use of mutants. Proc Natl Acad Sci USA 70: 817821

Wen JY, Kumar N, Morrison G, Rambaldini G, Runciman S, Rousseau J, van der Kooy D (1997) Mutations that prevent associative learning in C. elegans. Behav Neurosci 111: 354368

White JG, Southgate E, Thompson JN, Brenner S (1986) The structure of the nervous system of the nematode Caenorhabditis elegans. Phil Trans R Soc Lond B 314: 1-340

Wicks SR, Rankin CH (1996) The integration of antagonistic reflexes revealed by laser ablation of identified neurons determine habituation kinetics of the Caenorhabditis elegans tap withdrawal response. J Comp Physiol A 179: 675-685

(Received April 24, 2009 / Accepted March 17, 2010) 